\title{
Mapping of Some Neural Network Algorithms to a General Purpose Parallel Neurocomputer
}

\author{
Petri Kotilainen, Jukka Saarinen and Kimmo Kaski \\ Tampere University of Technology, Microelectronics Laboratory \\ P.O.Box 692, SF-33101 Tampere, Finland \\ e-mail petri@ee.tut.fi (Internet), tel. +358 31161 396, fax +358 31162620
}

The objective in our neurocomputer development project was to design a parallel general-purpose neurocomputer, which is capable to perform the network training as well as the forward mode operations. The modular structure and expandability would allow the system to be easily configured to meet various requirements of computation speed and system cost. The system is to be operated as a neural co-processor connected to a general-purpose computer.

The overall structure of our proposed architecture [1] consists of a number of identical processing units (PU), a tree-shaped network of communication units (CU) and a single host interface unit. The communication network is used to transfer the data between the processing units and the host interface using broadcast, read and write operations. Additionally the network can perform some global computation tasks. These tasks are the computation of the sum of all numbers sent from the processing units and finding the minimum or maximum values of the numbers sent by the PU's.

The most popular training method for multilayer perceptrons (MLP's) is the error back-propagation method [2], which consists of three phases, namely the forward phase, backward phase and weight updating phase. The computation of the forward phase can be performed by broadcasting one layer's input vector to all PU's, calculating the activation functions of the neurons on one layer in parallel in the PU's, reading the neuron outputs to the host interface unit and broadcasting them to all PU's as the input vector of the next layer. The computation of the backward phase can be performed by writing the error vector to the PU's calculating the delta factors within each PU and multiplying the delta vector with the transpose of the weight matrix. The communication network configured as an adder tree can be used for this purpose. These operations are repeated for each layer of the network. Finally, the weight updating can be performed in parallel within the PU's without additional communication between them.

Kohonen's Self-Organising Feature Map (SOFM) algorithm [3] is also suitable to be computed using the multiprocessor architecture. The most convenient way is to use neuron-parallel mapping by assigning one or more neurons to each PU. The recall phase of the trained SOFM involves broadcasting the input vector to all PU's and computation of the matching scores in parallel. The search of the best matching unit can be done by using the communication network configured for minimum search operation. In the training phase the index of the best matching unit is broadcast to all PU's, which can calculate the neighbourhood functions of the neurons of the network on the basis of this information. Finally the weights in the neighbourhood of the best matching unit are updated, which can also be performed in parallel.

Kanerva's Sparse Distributed Memory (SDM) [4] can be computed in the multiprocessor architecture by mapping one or more rows of the address and memory matrices to each PU. The calculation of the select vector in SDM involves broadcasting the address input vector to the PU's and computing the Hamming distances between the applied input vector and all rows in the address matrix. In the memory write operation the data input vector is broadcast to all PU's and added in parallel to the contents of each selected row of the memory matrix. In the memory read operation each PU calculates its own partial sum vector from the rows assigned to it. The final sum vector can then be calculated from the partial sums using the adder tree structure. The thresholding of the sum vector is then performed at the host.

Computation of the synaptic weighting factors and modifying the weights require most of the operations in the cases of multilayer perceptron networks and Self-Organising Feature Map. These operations can be effectively parallelised. In the case of Sparse Distributed Memory, the computation of the Hamming distances will become the most critical step in practice. In all three cases the communication delays between the host interface and the PU's depend mostly on the sizes of the vectors to be transferred and not so much on the number of PU's.

\section{References}

[1] P. Kotilainen, J. Saarinen and K. Kaski, "A Multiprocessor Architecture for General Purpose Neurocomputing Applications", Proceedings of $3^{\text {rd }}$ International Conference on Microelectronics for Neural Networks (MICRO-NEURO 1993), Edinburgh, Scotland (April 1993).

[2] R. Beale and T. Jackson, Neural Computing: An Introduction, Adam Hilger, Bristol (1990).

[3] T. Kohonen, Self-Organization and Associative Memory, Springer-Verlag, Berlin (1984).

[4] P. Kanerva, Sparse Distributed Memory, MIT Press, Massachusetts (1988). 